Recibido: 5 diciembre 2020 | Aceptado: 15 diciembre 2020 | Publicado: 23 diciembre 2020

Cita: Server Benetó, N. (2020). Caracterización discursiva de un género híbrido: la conversación humorística en «Nadie sabe nada». Normas, 10(1), 70-80. doi:http://dx.doi.org/10.7203/Normas.v10i1.19163

\title{
CARACTERIZACIÓN DISCURSIVA DE UN GÉNERO HÍBRIDO: LA CONVERSACIÓN HUMORÍSTICA EN NADIE SABE NADA
}

\section{DISCOURSIVE CHARACTERIZATION OF A HYBRID GENRE: HUMOROUS CONVERSATION IN NADIE SABE NADA}

\section{Natàlia Server Benetó}

The Ohio State University

Resumen

En los últimos años han surgido géneros (especialmente audiovisuales) que podríamos denominar híbridos, puesto que comparten características formales o funcionales de varios géneros tradicionales. En la línea de estas investigaciones, el objetivo de este estudio es la caracterización discursiva de un género híbrido con fuertes componentes conversacionales y humorísticos. Nuestro objeto de estudio ha sido el programa radiofónico de improvisación llamado «Nadie Sabe Nada», de la Cadena Ser, presentado por Andreu Buenafuente y Berto Romero. Dada la interacción aparentemente espontánea y no guionizada entre los presentadores, la pregunta de investigación es si se trata de una conversación coloquial o no y qué diferencias se encuentran con la conversación coloquial prototípica. Para ello, y desde la perspectiva de análisis de la conversación coloquial de Briz et al. (2002) y de la lingüística del humor de Ruiz Gurillo (2012, 2014), se han buscado características típicamente coloquiales en el corpus de "Nadie sabe nada» en el nivel fónico, pragmático, morfosintáctico, léxico y estructural para observar las semejanzas y diferencias entre la conversación y el género estudiado. Los primeros resultados muestran que este programa supone un género híbrido entre el monólogo de humor clásico y la conversación coloquial. Aunque los límites no sean taxativos, la intención comunicativa (transaccional) de los hablantes marca una línea clara entre la convesación coloquial prototípica y el discurso humorístico. Este género híbrido se ha denominado "conversación humorística».

Palabras ClaVE: Conversación; corpus; géneros discursivos; humor; análisis del discurso
Abstract

In recent years, genres have emerged (especially in the audio-visual field) that could be called hybrids, since they share the formal or functional characteristics of several traditional genres. In line with these investigations, the aim of this study is the discursive characterisation of a hybrid genre with strong conversational and humorous components. Our object of study has been the improvisation radio show called "Nadie Sabe Nada", broadcasted in Cadena Ser, presented by Andreu Buenafuente and Berto Romero. However, in some episodes, the radio show is structured around the two of them talking and interspersing topics of conversation that generate humour for 50 minutes. Given the seemingly spontaneous and unscripted interaction between both presenters, the subject matter is whether or not it is a colloquial conversation and what differences are found with the prototypical colloquial conversation. In order to do so, the perspectives of analysis of the colloquial conversation of Briz et al. (2002) and the linguistics of humour of Ruiz Gurillo $(2012,2014)$ have been taken into account. In doing so, the colloquial characteristics have been sought in the corpus of "Nadie sabe nada" at the phonic, pragmatic, morphosyntactic, lexical and structural levels in order to observe the similarities and differences between the two genres studied. Although the boundaries are not set, speakers' communicative intention (transaccional) sets a line between prototypical colloquial conversation and humorous discourse. This hybrid genre has been named "humorous conversation".

KEY WORDS: Conversation; corpus; discursive genres; humour; discourse analysis. 


\section{INTRODUCCIÓN}

El objetivo de este trabajo es identificar el género discursivo de un programa de radio, «Nadie Sabe Nada», presentado por Andreu Buenafuente y Berto Romero en la Cadena SER. Este programa presenta una fuerte hibridación, ya que, en apariencia, el intercambio desarrollado por los presentadores se plantea con una estructura que podría considerarse la de la conversación coloquial. La cuestión principal, por tanto, es determinar es si este programa pertenece al género de la conversación coloquial, aunque con gran presencia de humor, o si más bien es un producto humorístico como podría ser un monólogo o película de comedia, pero con las características formales y estructurales (a menudo, fingidas) de la conversación coloquial.

Con tal de abordar esta pregunta, se ha usado literatura de dos teorías principales: en lo que respecta a la conversación coloquial, la principal bibliografía ha sido la generada en el seno del grupo Val.Es.Co., a través de Briz (1996; 2002) y basándonos a su vez en García Ramón (2018). Por otro lado, para abordar la cuestión del humor, se han seguido los parámetros de Ruiz Gurillo (2014) sobre la lingüística del humor en español, basados a su vez principalmente en monólogos humorísticos.

Los resultados cuantificados y contrastivos, así como una fuerte basis cualitativa, nos han Ilevado a la conclusión de que el programa "Nadie Sabe Nada» constituye una conversación humorística, un género que no ha sido anteriormente estudiado ni planteado. En efecto, el humor se expresa a través de muchas otras manifestaciones discursivas, como la conversación coloquial, la entrevista o el debate. Para alcanzar tal fin, se pueden usar mecanismos de otros géneros y de otras modalidades, como se podrá observar en este estudio, pero consideramos que si hay una finalidad en mente que va más allá de la simple interacción social, por naturaleza propia esta intención ha de ser transaccional. El argumento se vuelve más fuerte cuando se analiza, nivel a nivel, cada una de las características presentadas por el programa, tanto en el área de la conversación coloquial como del humor.

\section{LA CONVERSACIÓN COLOQUIAL. PERSPECTIVAS TEÓRICAS}

Antes de desarrollar la teoría específica de este estudio, hemos creído adecuado repasar el modelo que hemos seguido en nuestro estudio y enumerar los rasgos que caracterizan la conversación coloquial de esta propuesta. Hemos seguido el resumen y planteamiento de García Ramón (2018: 29), basados en Briz y el Grupo Val.Es.Co (1995, 2010a). A continuación, repasamos estos rasgos, divididos en: rasgos primarios, rasgos propios del género y rasgos coloquializadores.

Rasgos primarios. Son aquellos referidos al registro, en este caso, el coloquial:

- Fin que persigue, interpersonal

- Tono informal

- Ausencia de planificación

Rasgos propios del género (conversación). Los rasgos prototípicos presentes en la conversación, ya sea coloquial o no, son los siguientes: 
- Oral

- Inmediatez o carácter actual

- Dinamismo

- Dialogal

- Alternancia de turnos no predeterminada

Finalmente, los rasgos coloquializadores forman parte de la coloquialización, es decir, el proceso mediante el cual se puede hablar de registro coloquial. La presencia de estos rasgos favorece que el registro sea coloquial. La novedad de Val.Es.Co al denominar el proceso de coloquialización radica en que, al ser un proceso, en el caso de que alguno de estos rasgos no esté presente, los otros actuarán de manera niveladora.

- Relación de igualdad social o funcional

- Relación vivencial de proximidad

- Marco de interacción familiar o cotidiano

- Cotidianeidad temática

\section{EL HUMOR: APROXIMACIÓN TEÓRICA}

Dado que el programa se define como «humor en estado puro», hemos creído necesario utilizar parámetros para acotar el género humorístico. Ruiz Gurillo (2012) defiende que la teoría general del humor de Attardo (2001) es la que más herramientas presenta para poder analizar los mecanismos del humor en diferentes productos humorísticos. La autora tiene en cuenta los recursos presentados por el autor y añade nuevos, los cuales se resumen en: estrategias narrativas, tipología textual, situación comunicativa, lenguaje y procesos inferenciales. De estos, los que más nos interesan para este estudio son los dos últimos.

- Lenguaje. Ruiz Gurillo argumenta que las elecciones en un texto humorístico no son «independientes ni aisladas, sino un hecho del uso del lenguaje» (Verschueren, 2002, 2009; Ruiz Gurillo, 2012: 78). El humor, por tanto, surge de unas elecciones conscientes por parte de los hablantes.

- Procesos inferenciales. Estos procesos facilitan el entendimiento de un texto como humorístico, ya que desencadenan el humor mediante las marcas e indicadores presentados por el lenguaje. Según Ruiz Gurillo (2014), el objetivo principal del humor, divertir, se consigue gracias a la «infracción de los principios pragmáticos que gobiernan la comunicación» (Ruiz Gurillo, 2014: 150). Estas infracciones son a los principios pragmáticos de Levinson (2000) y tienen como resultado numerosos mecanismos humorísticos. Para este estudio, los que más nos han interesado han sido las unidades fraseológicas, la variación (ámbito muy amplio) y los cuantificadores.

\section{Metodología}

El corpus principal de este estudio son dos episodios del programa radiofónico «Nadie Sabe Nada", retransmitido de 13 a 14h los sábados en la Cadena Ser y producido por El Terrat, que se inició en 2013. Los presentadores son Andreu Buenafuente y Berto Romero, dos cómicos, presentadores y actores catalanes. Aunque en la mayoría de 
programas los presendares van respondiendo preguntas que el público ha enviado previamente, sin haberlas leído, los episodios seleccionados para este estudio no cuentan con preguntas enviadas por el público, así que solamente participan los dos presentadores y algunas personas del público. Sin ninguna pregunta, los presentadores hilan temas y saltar de uno a otro, con lo que nuestra elección queda justificada. El programa también se graba en vídeo y se sube tanto a YouTube como a la página oficial de El Terrat; no obstante, pese a la multimodalidad del corpus, en este estudio solamente nos hemos centrado en la parte verbal, no en la paralingüística.

\section{ANÁLISIS}

\subsection{Rasgos situacionales}

Con el fin de caracterizar el programa, hemos analizado primero sus rasgos situacionales y después, los lingüísticos. En el primer paso, el análisis de los rasgos situacionales, nos hemos basado en Briz (1995) y García Ramón (2018), como se ha indicado en la introducción teórica. De todos estos rasgos, solamente nos hemos centrado en seis, explicados a continuación:

- Fin que persigue: interpersonal. La finalidad de una conversación coloquial es interpersonal, socializar, reforzar los lazos entre las personas. Cuando un texto es transaccional significa que tiene un objetivo concreto, que se quiere ver un resultado de la interacción. Ruiz Gurillo (2014: 39) defiende que el fin del humor es interpersonal. Sin embargo, en nuestra opinión, el humor tiene una finalidad muy clara, hacer reír a la audiencia, lo cual no coincide con la definición que se le da desde la teoría de Val.Es.Co. a la finalidad interpersonal. Como hemos visto anteriormente, hay un proceso de elección del lenguaje humorístico. Por lo tanto, si el emisor debe pensar qué mecanismos y recursos lingüísticos usar y esa elección y el resultado es lo que predomina en su uso del lenguaje, creemos que ya no se estaría hablando de una finalidad interpersonal sino transaccional. Aplicado a este estudio, creemos que se tomaría más una vertiente de finalidad transaccional; de hecho, en uno de los programas analizados, Berto Romero dice lo siguiente que el "Nadie Sabe Nada» es «hablar como si estuviesen en un bar, pero para que lo escuche todo el mundo".

- Ausencia de planificación. En línea con Briz (2001), concebimos la planificación como un espectro. Por tanto, aunque nuestro corpus no cuente con un guion como tal ni con una estructuración clara, hemos considerado que el hecho de que sea un programa de radio les condiciona a usar elementos extralingüísticos. A pesar de estos detalles, creemos que la falta de planificación es una característica lo suficientemente presente en los programas analizados del «Nadie Sabe Nada», si bien en menor medida que lo que sucedería en conversación coloquial.

- Carácter dialogal y alternancia de turnos no predeterminada. Como dice Briz (1995), esta característica es «una condición necesaria para que pueda hablarse de conversación» (Briz, 1995: 29). Creemos entonces que la toma de turnos en nuestro corpus no es predeterminada y que, además, se rige por una intención claramente transaccional dado que se usa como mecanismo para generar humor. 
- Relación vivencial de proximidad. La relación próxima de los interlocutores es un dato importante, ya que facilita que se transgredan las máximas conversacionales, lo cual facilita el humor, pero también afecta a otros aspectos de la conversación coloquial, como el grado de planificación.

- Marco de interacción familiar o cotidiano. Este punto tiene especial relevancia, ya que la conversación coloquial prototípica se suele dar en lugares no marcados. Sin embargo, el programa "Nadie sabe nada» se realiza en un estudio o teatro, delante de cámaras y micrófonos y ante un público asistente y durante un periodo de tiempo determinado, por lo que es un marco de interacción marcado. Aun así, esto no impide que se mantengan algunos de los rasgos situacionales típicos de la conversación coloquial.

\subsection{Rasgos lingüísticos}

Como se ha explicado en el marco teórico, la división realizada para analizar y contabilizar los rasgos lingüísticos del «Nadie sabe nada» ha seguido, con algunas modificaciones, la propuesta por Briz (1996). Por falta de espacio, seguiremos el mismo planteamiento que en el punto anterior y solamente comentaremos en este artículo los mecanismos que han sido particularmente relevantes para la conclusión del estudio.

\subsubsection{Nivel fónico}

- Alargamientos fónicos y pérdidas de sonido. Creemos que la presencia de estos mecanismos se puede deber a (1) la necesidad de rellenar espacios y de que no haya silencios; (2) la voluntad de mantener el turno de palabra para acabar la broma o hacerla más cómica, ya sea con o sin éxito; (3) las exclamaciones intensificadoras de actitud.

- Pronunciación marcada. Uno de los marcadores del humor propuestos por Ruiz Gurillo (2014: 157) era la variación, en el que se suelen incluir cambio de registro y de código lingüístico, pero también se podría incluir pronunciación marcada. Por lo tanto, en «Nadie sabe nada» encontramos ejemplos de pronunciación marcada tanto en la vertiente de la conversación coloquial, para intensificar lo dicho interpersonalmente, como en la vertiente del humor, para resaltar su carácter cómico.

\subsubsection{Nivel pragmático}

\subsubsection{Conectores pragmáticos}

- Control y regulación de contacto. La mayoría de estas partículas están insertadas en fragmentos «humorísticos» y parece ser que se usan para aumentar el efecto humorístico de la intervención, no tanto para regular el control.

(1) A: [asido] / eh // no que "haya sido" sino que está "asido" // [¿me entiendes o no?]

B: [explica- explícalo más] / que hay gente MUY idiota / [escuchando]

A: [vale, vale, vale] (risas) 


\subsubsection{Intensificadores (intensificación)}

La presencia de los intensificadores también es una constante común en el estudio de la conversación coloquial y de los géneros humorísticos, ya que se registra frecuentemente en ambos.

Morfológicos, sintácticos y léxico-semánticos. Hemos agrupado estos en el mismo punto porque creemos que no hay nada lo suficientemente destacable individualmente y porque hemos considerado que existe la misma intencionalidad en la mayoría de sus manifestaciones: romper la cortesía hacia el interlocutor y crear, así, humor.

(2) A: tú eres mu(y) listo / eh

B: no/ porque lo sé

A: uy qué tío más tontoo

\subsubsection{Atenuantes (atenuación)}

Diminutivos. En este estudio nos interesa saber en qué momentos se han utilizado con diminutivos y si se han empleado con fin humorístico en vez de para mantener la cortesía.

(3) A: sí sí / de un caballo que dijo / "ostia, qué bajita es la yegua" / [¿no?]

B: [ay] qué orejas / qué orejas más ricas tiene // qué orejitas más / [ricas]

Como podemos ver en el ejemplo, Berto Romero se «corrige» y opta por un diminutivo, lo cual parece ser un indicador de, ya que durante esa intervención tanto el público como ellos ríen y el presentador encuentra en el diminutivo una oportunidad para hacer aún más humor.

\subsubsection{Deícticos}

Cambio de código. Es habitual que los hablantes bilingües cambien de código en medio del acto comunicativo, pero, en nuestro corpus, ambos interlocutores tienen un manejo completo del español. Además, este cambio de código no se da al catalán, sino a otros idiomas, predominantemente inglés. Solo en una ocasión se hace uso del catalán, la cual es claramente humorística. En todas las otras ocasiones de cambio de código se usa el inglés, menos en una ocasión, bastante significativa, en la que se emplea el alemán.

(4) A: gràcies gràcies // ay gracias $(())$

$B$ : [risas] ¿qué pasa?

A: que he dicho "gràcies" en [catalán]

$B$ : [risas] [vale] pero sigue siendo una lenguaa [constitucional]

(5) A: ¿sabes esa marca que son solo animales? / ¿que los venden? / ME ENCAN[TA]

B: [síi] UNA ALEMANA

A: UH

B: UH

A: Schneiler

B: síi

A: scheider / [oo o Schisber]

$\mathrm{B}$ : [schimtz oo]

B: (()) [sí sí sí Scheiben Schmitz] no no / [Schmitz]

A: [Scheneder] / [Chamber] // [su]per caros 


\subsubsection{Nivel morfosintáctico}

\subsubsection{Deíxis personal}

Tú. Al analizar con más detenimiento los usos dados a este mecanismo, hemos detectado que suele emplearse para «atacar» al interlocutor, lo cual suele provocar humor, como hemos visto en otros fenómenos. Debemos tener en cuenta que ambos interlocutores tienen una relación vivencial muy próxima, por lo que estas trasgresiones no se consideran descorteses y menos aun cuando se piensa que están intentando hacer humor. En el caso del «Nadie sabe nada» esto no importan tanto porque se está haciendo humor.

Relaciones temporales y aspectuales. En primer lugar, hay una alta presencia de verbos formulados en presente, pero con valor de pasado o viceversa, especialmente los de habla, los cuales tienen directa relación con la alta presencia de relatos a lo largo de los dos programas. La importancia para nuestro estudio reside en que un texto escrito se suele seguir una línea temporal clara y los verbos concuerdan los unos con los otros, pero podemos comprobar que este no es el caso de los programas analizados. No obstante, esto no es suficiente para determinar si hay un guion previo o no, ya que los presentadores se podrían fácilmente adaptar a la situación y cambiar los tiempos sobre la marcha.

\subsubsection{Nivel léxico}

\subsubsection{Léxico}

Lexemas intensificados. Algunos ejemplos de léxico coloquial que entrarían dentro de esta categoría son «follón», «traumazo» o «chungo». Además, algunos parecen estar marcados sociolectalmente, como «chungo», «flipar» o «zurrar».

Frases hechas y expresiones metafóricas. Este es un recurso que coincide con los mecanismos del humor desarrollados por Ruiz Gurillo (2012; 2014), por lo que creemos que es clave tanto en la vertiente coloquial como en la humorística. Desde nuestro punto de vista, estas expresiones son una de las bases de la creación de humor en el programa porque aluden a la vida cuotidiana de los espectadores y porque pueden estar marcadas sociolectalmente, además de por quedar insertadas en secuencias humorísticas.

\section{Reguladores fáticos}

- Verbos de percepción. Creemos que el uso de estos verbos es una característica del propio presentador Andreu Buenafuente. Además del análisis cuantitativo de este estudio, dado que uno de los humoristas estudiados por Ruiz Gurillo (2012) era precisamente Andreu Buenafuente, hemos podido comprobar que este mecanismo forma parte de su idiolecto humorístico. Basándonos en el "Nadie sabe nada», creemos que estos verbos pueden usarse como mecanismo humorístico en el sentido que resaltan lo que se ha dicho, se lleva la atención del oyente a lo dicho previamente o lo que se dirá posteriormente, lo cual será humorístico. 
- Vocativos. Como lo anterior, hemos considerado que este mecanismo se usa para llamar la atención del interlocutor porque se ha dicho algo cuyo objetivo es humorístico. Por tanto, a a priori parecen usarse con el mismo valor que tendrían en la conversación coloquial, aunque en ocasiones sí parecen estar condicionadas por la situación humorística que les rodea.

\subsubsection{Nivel estructural}

Como se ha ido explicando a lo largo de estas páginas, una de las características que, a primera vista, podían indiciar que «Nadie sabe nada» es una conversación coloquial es, justamente, su estructura como conversación. Hay algunos parámetros que, a pesar de no pertenecer al nivel estructural en la bibliografía original, se han determinado más importantes para explicar la estructuración del programa y cómo esta se inclina más hacia una caracterización u otra.

Silencios. La importancia de los silencios en el programa radica en su análisis cualitativo, no cuantitativo. Como ya se ha mencionado, Ruiz Gurillo (2014: 160) considera que el aprovechamiento de las pausas es un mecanismo extralingüístico de creación del humor. Por tanto, creemos que es importante analizar los silencios de nuestro corpus en cuanto a su valor. Es por ello también que se han incluido en este nivel, el estructural, y no el que le correspondería, el fonético.

En los dos episodios analizados de «Nadie sabe nada» no se ha dado ninguna ocasión en la que haya habido más de 5 segundos de silencio, a no ser que fuese con una intencionalidad clara, las cuales explicaremos más adelante. Si los presentadores se encuentran ante una posible situación de silencio, recurren a conectores, adición de sonido, fórmulas de apertura o la música del programa para rellenarlos. Sin embargo, el programa no se puede permitir estar en silencio ya que están condicionados por el medio comunicativo y por la finalidad última del programa, hacer humor.

Como se ha introducido en el párrafo anterior, en este estudio hemos propuesto una posible clasificación de los silencios que se dan en el programa y de sus funciones pragmáticas y labores estructuradoras. Hemos clasificado estos silencios en cuatro categorías: las cuales repasaremos en las siguientes páginas. La primera categoría son los silencios en los que solamente ríe el público y los presentadores callan.

(6) B: [peeroo]

A: porque (risas) te- / ahora te estoy incomodando / ¿no?

En segundo lugar, los silencios de creación de expectativas ocurren cuando los presentadores callan o hacen pausas para crear más «tensión» humorística con respecto a lo que están diciendo, lo cual también coincide con el aprovechamiento de las pausas de Ruiz Gurillo (2014).

En tercer lugar, y basándonos en Méndez Guerrero, también hemos anotado presencia de silencios estructuradores, cuya función es responder «a reglas o principios que organizan o estructuran la conversación» (Méndez Guerrero, 2014: 71) y se les atribuye la función de cambio de tema, dinamizar la conversación, mostrar petición de apoyo o rechazo, entre otros. 
La cuarta clasificación que se podría dar a los silencios del «Nadie sabe nada» es la de silencios discursivos. Según Méndez Guerrero su papel es «señalar la función comunicativa que se está expresando en la interacción», entre las cuales se incluye «disentir, asentir, mostrar acuerdo, afirmar, atenuar, intensificar, reformular, ironizar» (2014: 71).

Solapamientos y habla inmediata. Se ha considerado adecuado estudiar estos dos parámetros de manera conjunta porque el programa está caracterizado por ambos mecanismos. Así, los cambios de turno en nuestro corpus se dan, por orden, mediante solapamientos, habla inmediata o silencios.

Creemos que los solapamientos se usan habitualmente como mecanismo humorístico, especialmente en ciertas intervenciones. Estas elecciones pueden ser porque (1) quieren seguir añadiendo material cómico a su intervención y ven que una posible manera de llevarlo a cabo es interrumpiendo a su interlocutor, aunque se desvíen del tema, (2) hablan al mismo tiempo y no se escuchan, algo que no ocurriría con tanta frecuencia en una conversación coloquial, o (3) la risa propia les impide intervenir en su turno e interrumpen al interlocutor.

Continuación, cierre y cambio de tema. Desde nuestro punto de vista, la menor presencia de estos mecanismos en nuestro corpus es debido a que el número de temas que se dan es mucho más elevado en el programa de «Nadie sabe nada» que en una conversación coloquial prototípica. Asimismo, la duración de cada tema es menor que la que suele tener en la conversación coloquial porque hay mucho más dinamismo: no se pretende profundizar personalmente en lo que se habla, sino que resulte lo suficientemente interesante para que sea humorístico. En esta línea, no parece importar tanto que los temas no se concluyan, que las interacciones se solapen o que no se preste excesiva atención a lo que se está diciendo mientras que se hable y se haga humor.

Relatos de voces. En ambos programas hay una gran presencia de relatos de voces, lo cual creemos que se debe a que estos son el eje estructurador del programa. Dentro de esta sucesión de cosas que se cuentas los presentadores y el público (cuando interviene), hay ocasiones en las que dramatizan. En estos fragmentos, los interlocutores se limitan a relatar las voces de las personas a las que están «interpretando», sin fingir ninguna voz ni ningún acento. En otras ocasiones, sin embargo, optan por emular completamente a la persona a la que están imitando, cuya intención puede ser, o bien darle más realismo al relato, o bien hacer humor.

Fórmulas de cierre enumerativo. Desde nuestro punto de vista, es difícil acotar los cierres completos de tema en la conversación coloquial, pero aún ha resultado más difícil hacerlo en los programas analizados ya que la velocidad a la que se cambia de tema es mayor que en una conversación coloquial prototípica. Por ello, no creemos que las fórmulas de cierre enumerativo sean una característica relevante en el «Nadie sabe nada» por lo que ya se ha mencionado anteriormente. En vez de ello, los temas se van enlazando por ocurrencias propias de los hablantes, las cuales no suelen estar muy a menudo reflejadas lingüísticamente. 
Tras este análisis del nivel estructural, creemos adecuado señalar que «Nadie sabe nada» se percibe dentro del gradiente propuesto por Briz (1996) como un texto que, estructural y lingüísticamente, parece presentar las características de la conversación coloquial, pero que formalmente se asemeja más a un debate político público que a una conversación coloquial prototípica. Lo que mayor importancia toma en estos casos es el destinatario último del discurso: en una conversación coloquial, dado que la intencionalidad es interpersonal, se entiende que A es el destinatario de B y viceversa (más participantes si los hay y todos recíprocos). No obstante, en un debate público, aunque los interlocutores estén hablando entre ellos, se podría decir que solo le interesa lo que pueda decir el otro si le puede beneficiar en su argumento para poder convencer a un público, a una audiencia. A y $\mathrm{B}$ pueden debatir sobre qué medidas económicas adoptar, pero sus respuestas no están percibidas para estrechar sus lazos, sino para que $\mathrm{C}$, una audiencia, los escuche y decida su voto final. Por tanto, esta es otra diferencia estructural con respecto a la conversación coloquial prototípica.

\section{CONCLUSIONES}

Tras realizar el análisis mediante la teoría de la conversación coloquial de Briz y el grupo Val.Es.Co y las teorías de caracterización del humor en español de Ruiz Gurillo, y como ya se ha comentado al principio, se ha determinado que el programa «Nadie sabe nada» podría considerar una conversación coloquial periférica, bautizada como «conversación humorística». El programa presenta las características básicas de una conversación y aunque presente algunas de las características del registro coloquial (tono informal, cierta ausencia de planificación) y de la coloquialización (relación de igualdad social o funcional, relación vivencial de proximidad o cotidianeidad temática), no tiene intencionalidad interpersonal, sino transaccional (crear humor) y el marco de interacción no es cotidiano (es la radio, lo cual conlleva cambios en la estructura y las interacciones).

Por lo tanto, es una conversación cuyo objetivo último es crear humor. El programa presenta características lingüísticas que coinciden con la conversación coloquial prototípica a través de los diferentes niveles lingüísticos, pero su presencia no es suficiente para determinar una clasificación concluyente. Este estudio ha tomado una aproximación cuantitativa en algunos casos, pero, sobre todo, los parámetros lingüísticos se han analizado cualitativamente. Con todo, se ha podido llegar a la conclusión de que la mayoría de estos mecanismos, aunque prototípicos de la conversación coloquial, no siguen su misma intencionalidad y, por tanto, no serían realmente característicos de la conversación coloquial, sino de una conversación humorística.

El «Nadie sabe nada» no es el único programa que puede dar lugar a estudios: la sección El consultorio de Berto del late-show Late Motiv está a medio camino entre planificación absoluta e improvisación. Asimismo, otro programa conversacional y humorístico alejado del formato entrevista podría ser La vida moderna, y otros programas de formato entrevista pero que pueden llegar a saltarse las reglas de esta y llegar a ser una conversación son La Resistencia, No te metas en política e incluso las propias entrevistas de Andreu Buenafuente a sus invitados. 


\section{BIBLIOGRAFÍA}

Briz Gómez, Antonio (2001): El español coloquial en la conversación. Esbozo de pragmapagmática, L'Hospitalet de Llobregat, Ariel Lingüística.

Briz, Antonio (1996): El español coloquial: Situación y uso, Madrid, Arco Libros.

Briz, Antonio y Grupo Val.Es.Co. (1995): La conversación coloquial. (Materiales para su estudio), València, Universitat de València Publicacions.

Briz, Antonio y Grupo Val.Es.Co. (2002): Corpus de conversaciones coloquiales. Anejos, Madrid, Arcos Libros.

García Ramón, Amparo (2018): Epistemicidad en interacción: (a)simetrías epistémicas en secuencias de acuerdo y su relación con la construcción de roles funcionales en conversaciones y entrevistas (Tesis doctoral), València, Universitat de València <http://roderic.uv.es/handle/10550/69401>.

López Serena, Araceli (2014): «De la oralidad fingida a la oralidad simuladora de realidad. Reflexiones en torno a la coloquialización del discurso como estrategia mediática», Español Actual, 102, 37-75.
Méndez Guerrero, Beatriz (2013): «El silencio en la conversación española. Reflexiones teórico-metodológicas", Estudios interlingüísticos, 1(1), 67-86.

Ruiz Gurillo, Leonor (2012): La lingüística del humor en español, Madrid, Arco Libros.

Ruiz Gurillo, Leonor (2013): «El monólogo humorístico como tipo de discurso. El dinamismo de los rasgos primarios", Cuadernos AISPI, 2, 189-212.

Ruiz Gurillo, Leonor (2013): «Narrative strategies in Buenafuente's humorous monologues» en Alvarado Ortega, María Belén y Leonor Ruiz Gurillo, eds., Irony and Humor: From Pragmatics to Discourse, Amsterdam, John Benjamins Publishing, 107-140.

Ruiz Gurillo, Leonor (2014): «Infiriendo el humor. Un modelo de análisis para el español», Revista CLAC (Círculo de Lingüística Aplicada a la Comunicación), 59, 148-162.

\section{Recursos electrónicos}

Página web del programa Nadie Sabe Nada https://elterrat.com/radio/nadie-sabe-nada/ <Consultada: 25/01/2019> 